\title{
Continuous robot control using surface electromyography of atrophic muscles
}

\author{
Jörn Vogel ${ }^{1}$, Justin Bayer ${ }^{2}$ and Patrick van der Smagt ${ }^{2,3}$
}

\begin{abstract}
The development of new, light robotic systems has opened up a wealth of human-robot interaction applications. In particular, the use of robot manipulators as personal assistant for the disabled is realistic and affordable, but still requires research as to the brain-computer interface.

Based on our previous work with tetraplegic individuals, we investigate the use of low-cost yet stable surface Electromyography (sEMG) interfaces for individuals with Spinal Muscular Atrophy (SMA), a disease leading to the death of neuronal cells in the anterior horn of the spinal cord; with SEMG, we can record remaining active muscle fibers. We show the ability of two individuals with SMA to actively control a robot in 3.5D continuously decoded through sEMG after a few minutes of training, allowing them to regain some independence in daily life. Although movement is not nearly as fast as natural, unimpaired movement, reach and grasp success rates are near $100 \%$ after $50 \mathrm{~s}$ of movement.
\end{abstract}

\section{INTRODUCTION}

SH and SV are two of the approximately $0.1 \%$ of the European population suffering from SMA (Spinal Muscular Atrophy): a disease leading to the death of neuronal cells in the anterior horn of the spinal cord. This disease, which they have carried with them since birth, has lead to a situation where their limb movement is limited to minute thumb abduction, allowing them to control their wheelchair. That gives them some freedom to move around; but as they cannot move their arms or legs, every other physical interaction with their environment must be realised through human assistants. Despite their wheelchair control, they cannot go outside alone: as the doorbell as well as the elevator button must be pressed to be operated, they have no chance to re-enter their home autonomously. Let alone autonomous drinking.

The development of lightweight robots can profoundly change the field of assistive robotics for physically disabled individuals. Simple environmental interaction chores can be easily realized by such systems, especially when guided by its user through a brain-computer interface (BCI).

But BCI are still in an infant state. First, interpreting biological data for $\mathrm{BCI}$ is a topic of research: the corresponding biological source signal contains time-coded data represented by large number of neurons/nerves/muscle fibers, but usually only a very small subset of these are recorded, leading to nonstationary behavior. Second, their application

\footnotetext{
${ }^{1}$ Jörn Vogel is with the Institute of Robotics and Mechatronics, German Aerospace Center, D-82234 Oberpfaffenhofen, Germany joern. vogel at dlr.de

${ }^{2}$ Justin Bayer and Patrick van der Smagt are with the Institute for Informatics, Technische Universität München, D-80333 München, Germany bayer.justin at gmail.com

${ }^{3}$ Patrick van der Smagt is also with TUM-fortiss, Guerickestr. 25, 80805 Munich
}

to the patient is involved. Invasive methods lead to promising results [1], [2], [3], [4], [5] and have indeed been used for 3D robot arm control [6], [7]. Nonetheless, such methods remain heavily debated with respect to their associated discomfort and risk for the user, while signal interpretation is highly variable. Indeed, invasive methods are generally used as a "last resort", when noninvasive methods cannot be applied. But also noninvasive methods require mounting sensors on the body, which is very complex and time-consuming for Electroencephalography (EEG), but it also requires thirdparty assistance when using surface EMG sensors for the physically disabled. A promising approach is the use of eye tracking systems. Unresolved challenges include that extraction of more than 2D is very noisy [8] and not realistic beyond that. Thus, eye tracking alone is not suited as a robot control interface currently.

Of the aforementioned BCI methods, surface EMG can be considered the least cumbersome. Such EMG systems are affordable and electrode placement is fast; its signal, involving amplification factors by three orders of magnitude only, is relatively stable. We have previously reported the use of EMG for arm position [9] reconstruction, and its stability inspired us to attempt similar methodologies for individuals with nonfunctional remaining muscle activity.

The use of surface EMG for prosthetic robotics is nothing new. The initial ideas stemmed from prosthetic hand robotics, and various publications on the matter [10], [11], [12], [13], [14] show that EMG can serve as an excellent means to control dexterous hand prostheses. Also arm position reconstruction can be done through EMG, either using models of the arm geometry [15], [16], [17] or without [9]. The latter approach, which controls the position and orientation of the robot end-effector in 6D, leads to a nonnegligible error in absolute end-effector positioning, but by controlling endeffector velocity rather than position [18] the system can be used with considerable accuracy to grab objects.

In this paper we aim to exploit a similar interface to control a robot by two 45-year old individuals with Spinal Muscular Atrophy (SMA) type IIa. This disease leads to death of motor neurons in the spinal cord, such that the corresponding muscle fibers can no longer be activated and degenerate. In effect, after many years of progressive disease no voluntary limb movement is possible, with the exception of, in one of the individuals, minimal thumb movement with which a miniature joystick can be pushed to control a wheelchair. For this individual, thumb movement does not suffice for 3D robot control; for the other, who can not operate a joystick at all, sip-and-puff control is used to steer the wheelchair. 


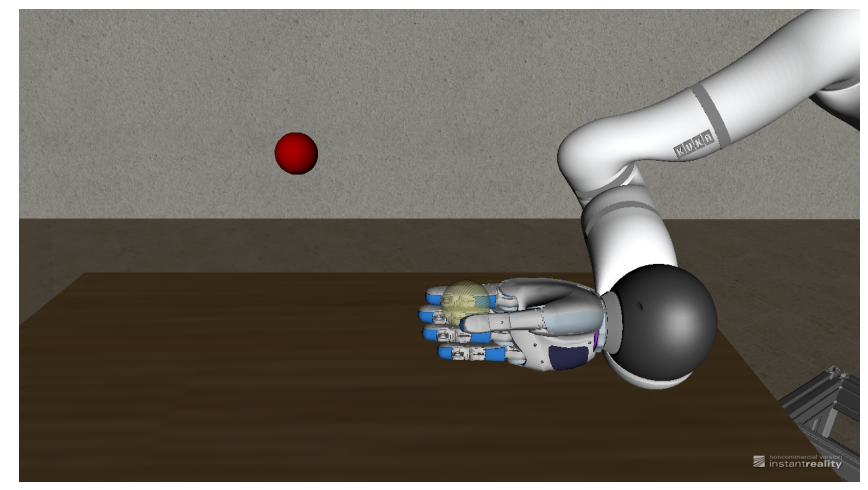

Fig. 1. Robot visualization as presented to the subject during the tasks. The red sphere in the background illustrates the target location, the semitransparent off-white sphere serves for highlighting the robot end-effector position.

Our goal is to develop an sEMG-based interface through which these individuals can control a robotic arm for simple grasp and move operations. To this end, we exploit the EMG signal stemming from residual arm muscle fibers, which can be voluntarily activated but which do not suffice to move the limbs.

\section{APPROACH}

In the experiments presented in this work we decode continuous control signals in three Cartesian degrees of freedom and a discrete grasp trigger from surface EMG signals measured on the upper limb of two subjects who suffer from SMA. The decoded control signals are used to operate a full dynamic simulation of a robotic system in a 3D virtual environment (see Figure 1).

In the following description we refer to experimental sessions as a combination of training phase and assessment phase. In the training phase, data is recorded in order to train a neural network as a decoder for the needed control signals. In the assessment phase, multiple trials are conducted in order to evaluate the performance of a specific decoder. A trial consists of an arbitrary number of reaching tasks that the subject has to perform with the simulated robotic system. The parameterization of the neural network decoder stays unchanged within the assessment phase.

\section{A. Data acquisition and preprocessing}

Surface EMG signals are composed of the superposition of activity of multiple underlying muscle fibers. More precisely it is the superposition of motor unit action potentials (MUAP). The amount of MUAPs correlates to the force the muscle is exerting, and following from the superposition, so does the sEMG amplitude. In our setup, muscular activity is recorded using Delsys ${ }^{\circledR}$ Trigno wireless surface EMG Electrodes. The Trigno system provides an analog differential signal in the range of \pm 5 Volts which is bandpass filtered between $20-450 \mathrm{~Hz}$ and sampled at $2 \mathrm{kHz}$. EMG data is digitized using a National Instruments ${ }^{\mathrm{TM}}$ NI 9144 EtherCAT slave chassis with NI 9205 16-bit analog input module. The EtherCAT slave chassis is connected to a VxWorks real-time computer which serves as the EtherCAT master and acquires the digitized raw-EMG signals at a $1 \mathrm{kHz}$ rate. This real-time system is also used to evaluate our preprocessing/filtering methods in order to extract the feature vector, which in our case consists of the amplitude of the sEMG signal.

A common approach to extract a smooth amplitude signal is the filtering of sEMG data employing low-pass filters; channel-wise moving averages based on time windows or with an exponential decay yield good results. Band-pass filters are commonly used as well. Removing high frequencies leads to two undesirable effects however. First, a delay is induced (due to window size and filter order) which is unwanted for online control applications. Second, sudden bursts or spikes are essentially flattened out. This is due to the nature of low-pass filtering and further reinforced by the shape of MUAPs. A common and crucial step, the full wave rectification can partially overcome these difficulties: the differential sEMG signal is transformed to its absolute value. Alas, the spike is a very local phenomenon while its effect on limb movement extends over a longer period because of movement inertia. After evaluation of a range of filters which we thought well suited to the problem, including median filters, wavelets, various Fourier transforms and window based or exponential moving averages, we found that a filter specifically customized for the characteristics of sEMG data and the task of posture estimation was beneficial.

Ideally, such a filter needs to (a) preserve the information contained in spikes for a longer time and (b) be relatively smooth. Our approach consists of the following stages which we apply to each channel separately:

- subtract mean;

- normalize to \pm 1 amplitude;

- full wave rectification;

- moving max filter (window: 50 samples);

- Savitzky-Golay filter (window: 500 samples, degree: 5). The mean and the normalization factor are extracted from a training data set and kept constant within an experimental session. After the full wave rectification of the signal $x_{1: T}$, we slide a time window of size $k$ over the signal and select the maximum value from it:

$$
\tilde{x}_{t}=\max \left|x_{t-k: t}\right|,
$$

which will result in a step-like signal. In order to obtain a smooth signal, we employ a local least-squares filter by fitting polynomial at each time step. While this might seem costly at first, [19] showed that efficient computation is possible. The filtering can not only be performed by solving a least-squares problem and subsequent evaluation; alternatively, it can be computed as a linear combination of the values at the preceding time steps of the signal,

$$
\hat{x}_{t}=\sum_{l=1}^{L} \alpha_{i} \tilde{x}_{t-l},
$$

where the set of coefficients $\left\{\alpha_{i}\right\}$ is independent of the input data and only depends on the parametrization of the filter: the window size $L$ and the degree of the polynomial $D$. For an 


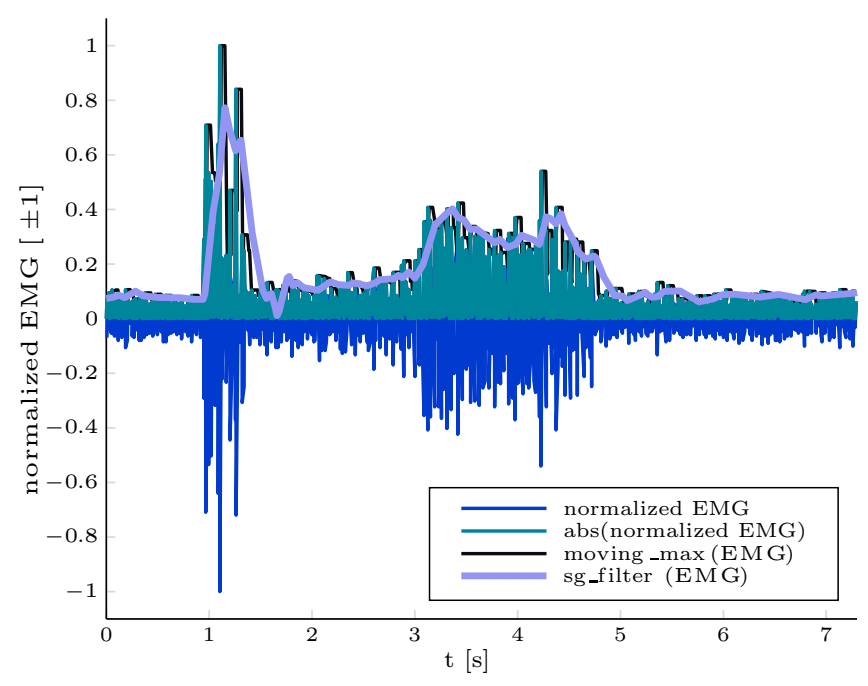

Fig. 2. Illustration of the single stages of the sEMG preprocessing tool chain on an exemplary data set.

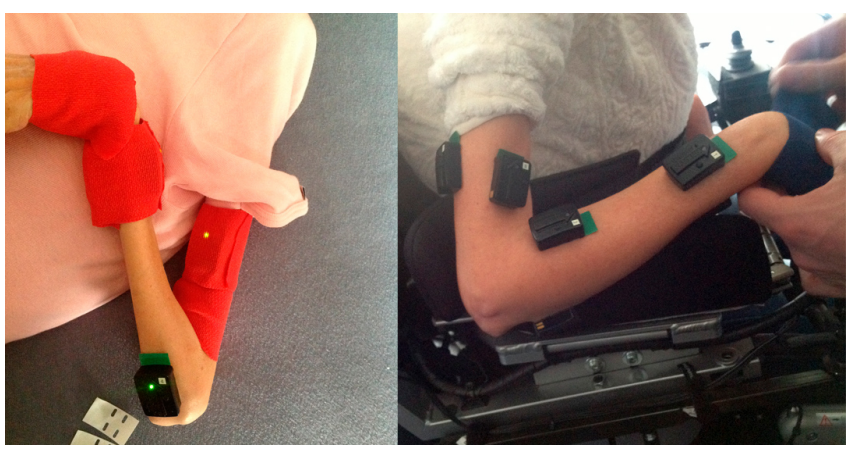

Fig. 3. Electrode placement for subject SH (left) and SV (right). Electrodes are fixed with doublesided medical tape and additionally wrapped with medical cohesive tape to ensure fixation (left).

illustration of the process see Figure 2. This finally provides a comparably smooth hull of the EMG-amplitude, which is used as a feature for decoding the control signals.

\section{B. Electrode configuration}

In the experiments conducted, we equipped subject SH's left arm with 6 and subject SV's right arm with 7 sEMG electrodes, respectively. Electrode placement was chosen based on visual (qualitative) signal inspection. We sampled different locations for each electrode in areas on the arm where the subjects reported to be able to feel muscle bulging. When activity was notable on the live view of the sEMG signal, we attached the electrode to that position with doublesided medical grade adhesive tape. To ensure fixation of the electrodes, we additionally wrapped them with cohesive medical tape. Table I lists the muscle anatomically most closely located to the single electrodes, based on the physiology of the healthy arm. It has to be noted that due to the advanced muscular atrophy it can not be guaranteed that these correspond to the muscles we actually recorded from. Figure 3 shows the placement for some of the electrodes for both subjects.
TABLE I

ELECTRODE PLACEMENT

\begin{tabular}{|c|c|c|}
\hline $\begin{array}{c}\text { Electrode } \\
\text { Nr. }\end{array}$ & $\begin{array}{c}\text { Muscle in proximity } \\
\text { to Electrode in SH }\end{array}$ & $\begin{array}{c}\text { Muscle in proximity } \\
\text { to Electrode in SV }\end{array}$ \\
\hline 1 & flexor pollicis & extensor digitorum \\
2 & extensor digitorum & anconeus \\
brachioradialis \\
3 & flexor carpi radialis & anconeus \\
4 & flexor carpi radialis \\
5 & biceps brachii & flexor pollicis \\
6 & triceps brachii & triceps brachii \\
7 & - & biceps brachii \\
\hline
\end{tabular}

\section{Simulated robotic system}

The long term goal of this experiments is to provide control over a robotic hand-arm system to people with physical disabilities, using a surface EMG interface. The work presented here serves as a groundwork to this goal. To validate the feasibility of the approach, we tested the EMGcontrol interface on a full dynamics simulation of the DLR Light-Weight Robot III (LWRIII) [20], [21] in combination with the DLR 5-finger robotic hand [22]. Combined with its soft-robotics control schemes, the LWR [23], [24] has shown its applicability to safe human robot interaction [25] and as an assistive robotic device in multiple scenarios [6].

The robot simulations virtual environment is displayed on a Samsung UE46ES6300 46inch 3D LED monitor. To enhance depth perception in the visualization of the robot simulation we make use of the 3D rendering capabilities of the instantreality virtual reality framework [26]. Subjects have to wear active shutter glasses to perceive the stereoview effect (see Figure 4). The visible scene (Figure 1) is composed of the robot positioned close to a table above which target spheres appear in order to be reached by the subject. A semi-transparent sphere indicates the location of the robots end-effector position.

\section{Training and signal decoding}

To gather ground truth data for system training, we use a paradigm based on visual stimuli. In the training phase the robot executes a so-called "center out and back" motion along the cardinal axes. The subject is asked to monitor this
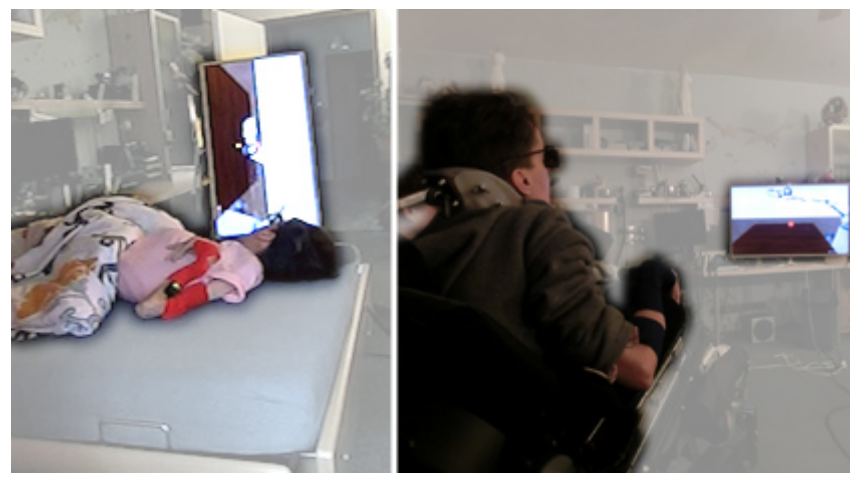

Fig. 4. Participants SH and SV equipped with sEMG electrodes and shutter glasses, while controlling the robot simulation on a 3D-monitor One participant preferred to lie down during the experiments, whereas the other participant preferred to control the robot sitting in the wheelchair. 
TABLE II

CARDINAL AXIS AND CORRESPONDING DIRECTION FOR THE USER.

\begin{tabular}{|c|c|c|c|}
\hline Cardinal Axis & Direction & Cardinal Axis & Direction \\
\hline$+X$ & right & $-X$ & left \\
$+Y$ & away & $-Y$ & towards \\
$+Z$ & up & $-Z$ & down \\
\hline
\end{tabular}

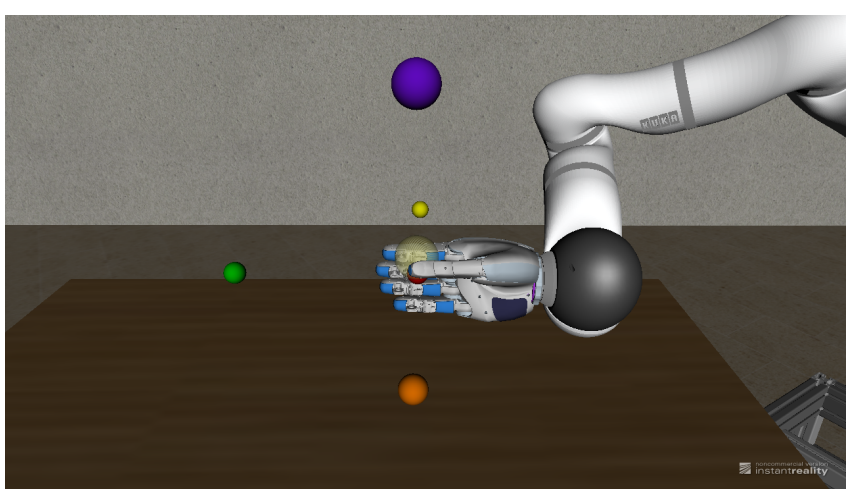

Fig. 5. Target placement during training phase. In this still picture, the robot is about to move to the highlighted purple target, located at the $+Z$ direction. The $+X$ target is hidden within the structure of the robot, but it becomes visible, when highlighted.

motion and perform muscular activation that shall be associated with the observed movement direction. Starting from a center position, the cardinal axes correspond to directions for the user as summarized in Table II. The target spheres for the training phase are located along these directions in a distance of $0.25 \mathrm{~m}$ from the center target (see Figure 5). As the $-Z$ target would be located underneath the table surface in the used target configuration, this target is omitted. The training phase typically consists of multiple repetitions of a training block. In the experiments presented here, a training block consists of two repetitions of all movement directions in random order. The target is visually indicated before the movement begins, and EMG is recorded during and after movement. To acquire equally many repetitions for each direction, the upper target is presented twice within one cycle, as the corresponding down target is missing. To gather training data for the discrete grasping signal, the robotic hand performs a grasping motion every time the robot reaches the center target.

From the data collected in the training phase, the EMG mean and normalization factor are extracted (see Section II-A), and a neural network (NN) is trained to decode desired end-effector velocity and a grasp activation trigger from the EMG data. In the initial set of experiments presented here, only data from one training block are used for training the NN.

Using the MathWorks ${ }^{\circledR}$ Matlab Neural Network Toolbox, we train a deep neural network consisting of two nonlinear hidden layers. The hidden layers consisted of 10 units each, and tansig was used as the transferfunction. We typically trained the neural network with randomly resampled data points and $\sim 10$ training epochs using Levenberg-Marquardt optimization.

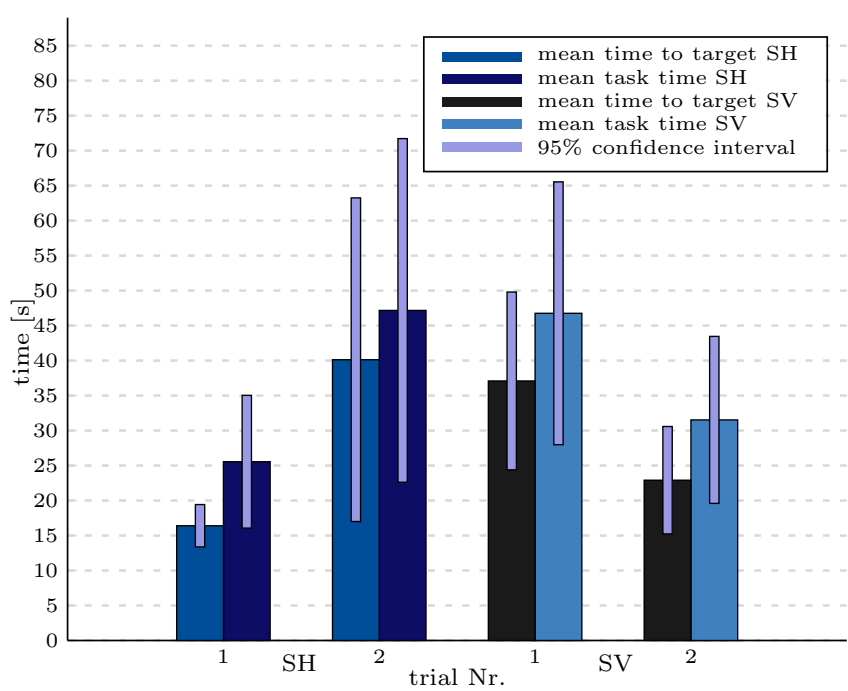

Fig. 6. Average times to get within the target diameter (left bar in each pair) and to complete a task (right bar in each pair) per trial.

\section{EXPERIMENTS AND RESULTS}

To evaluate the performance of the decoder, we asked the subjects to perform a number of grasp tasks. In this task we show the target at a point randomly chosen but $30 \mathrm{~cm}$ away from the current robot position. The task is to grasp the target object, meaning to place the hand within $3 \mathrm{~cm}$ of the target and evoke a grasp action. To clarify when a target is acquired, the task state (moving, at target, grasped) was shown by coloring the target (red, yellow, green) correspondingly; distinguishable beeps indicated the change of task state. At the end of a task, the robot is automatically positioned at the center of the target area and, after a 3 second pause, the next task is started automatically.

In this initial set of experiments the participants performed 2 trials, each of which contained 30 target tasks. We did not

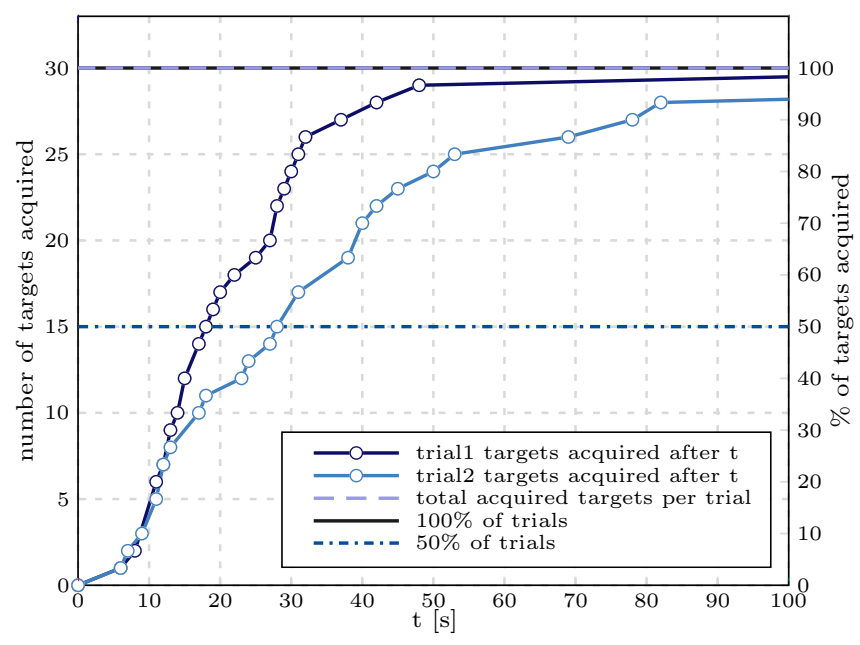

Fig. 7. Trial time analysis for 2 trials with 30 targets each, performed by subject $\mathrm{SH}$. Depicted is the number/percentage of targets successfully reached dependent on a time-limit $t$ for each single target in seconds. For simplicity $t$ is limited to $100 \mathrm{~s}$, the longest time for a single target was $t=363 \mathrm{~s}$. 


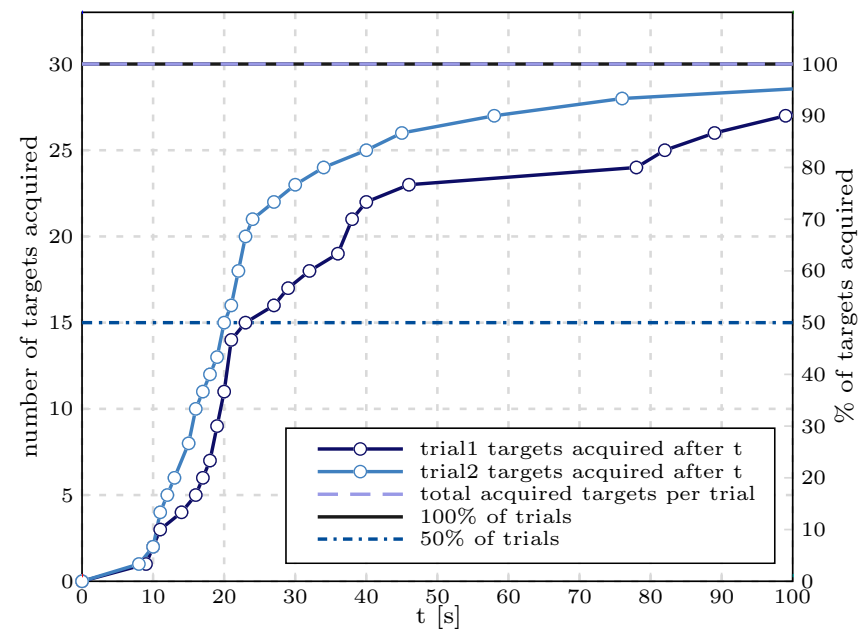

Fig. 8. Trial time analysis for 2 trials with 30 targets each, performed by subject SV. Depicted is the number/percentage of targets successfully reached dependent on a time limit $t$ for each single target in seconds. For simplicity $t$ is limited to $100 \mathrm{~s}$, the longest time for a single target was $t=261 \mathrm{~s}$.

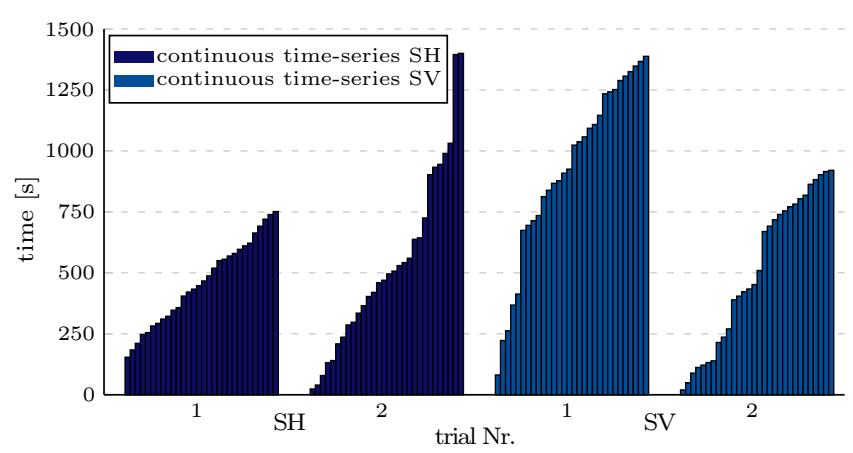

Fig. 9. Continuous time series analysis (inter task pause times are omitted) for 2 trials with 30 targets each, performed by subjects SH and SV. While the longer task times in trial 2 of subject SH may be an indication of fatigue, no such effect is evident for subject SV

specify a time limit at which a single task was aborted. Figure 6 depicts the mean times over all tasks for the single trials. For each pair of bars, the left one shows the average time needed to first reach the target, while the right bar shows the average time for task completion, i.e. evoking a grasp at the target location. Differences are caused by the fact that evoking a grasp takes some time and additionally, in some cases, grasp may be decoded as movement, and the participant must reposition the robot.

Figure 7 shows a more detailed trial time analysis of the two trials performed by subject SH; Fig. 8 for SV. Some tasks last several minutes; but for half of the targets the completion time was less than $30 \mathrm{~s}$ each. Looking at the time series analysis depicted in Fig. 9 it can be seen that for subject SH performance degrades at the end of the second trial, which may be an indication of fatigue. For subject SV no such clear evidence is found in the trial times. Another reason for longer task times is evident in the direction the goal is located. Fig. 10 shows the direction dependency of the decoder, with respect to task completion time: movement in

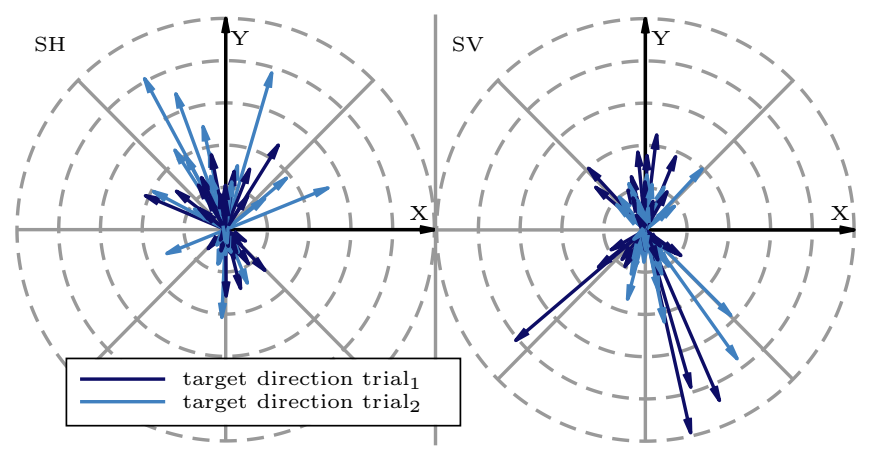

Fig. 10. Dependency of the task execution time on the target direction (in the $X-Y$ projection plane). The single vectors correspond to the target direction scaled with the task execution time. $X-Y$ projection is chosen, as no dependency on the $Z$ direction is noticeable. For trial 2 of subject SH (left) targets in the $+Y$ direction take longer. For both trials of subject SV (right) targets in the combined $+X /-Y$ direction require longer times.

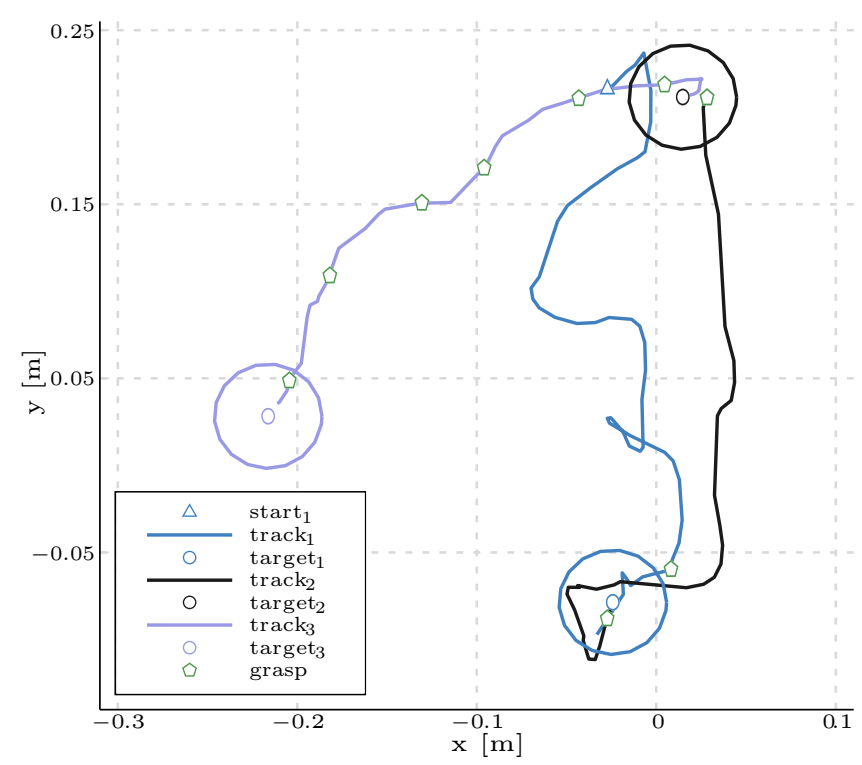

Fig. 11. Top-down projection of three exemplary chosen consecutive targets from one trial of subject SV. Execution times for these targets were $9.8 \mathrm{~s}$, $11.0 \mathrm{~s}$, and $7.6 \mathrm{~s}$, respectively.

certain directions are "more difficult" to make and therefore take longer. Example trajectories are shown in Figure 11. It is clear that the corresponding trajectories are not Cartesian straight; the participants have to continuously correct the movement to reach the target.

Another evident issue can be noticed in track ${ }_{3}$, which shows a repeating sequence of grasps during movement. These false grasp activations occur in many of the tasks, as can be seen from Figure 12. Depicted is the average number of false grasps during movements up to $60 \mathrm{~s}$. For this, grasp triggers decoded while the robot hand is outside the target by $4 \mathrm{~cm}$ are counted as false grasps. On the one hand it is noticeable that the amount of false positive grasps rises in trial number 2 for both subjects, which may be a cause of fatigue. On the other hand, subjects were not instructed to prevent occurrence of grasp commands away from the target location. Therefore, the peculiarity of false positive grasps 


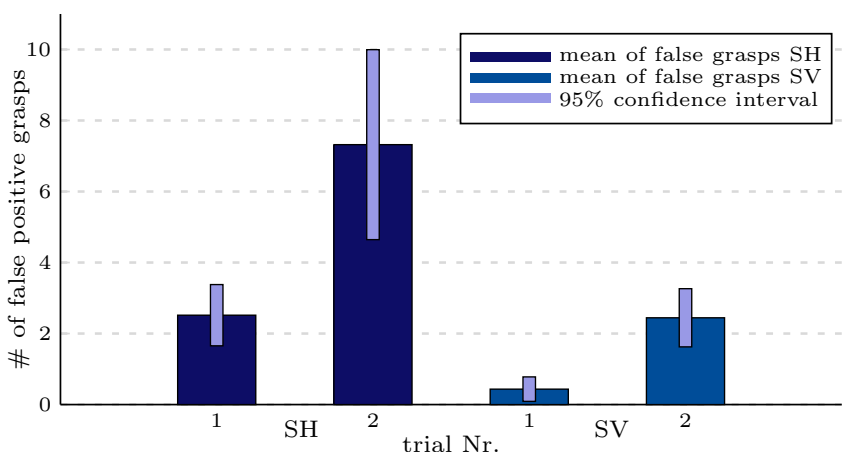

Fig. 12. Average false positive grasps (in tasks shorter than 60 seconds) over trials for subject SH and SV. Performance of the grasp decoder decreased noticeably in the second trial for both subjects.

has to be investigated in future experiments.

\section{CONCLUSION}

The preliminary results presented in this report originate from the very first set of experiments conducted within this study. Except for a short exploration time, the subjects, who had never controlled a robot through an EMG-based interface, were basically untrained on the system. Yet the results show that both subjects could achieve functional control over the simulated robotic system via the sEMG interface after very short training times. SV, who controls her wheelchair over a 2D discrete joystick, reported that the muscular effort needed to control the robot in 3D space was not more intense than for her wheelchair control. Furthermore the participant remarked to be not able to use a joystick providing 3 degrees of freedom. Likewise, subject SH, who can no longer use a mechanical interface like a joystick at all, described the control effort as minimal.

The decoding techniques used in this experiment are refinements of our previous work on sEMG robot interfaces for unimpaired subjects [9]. The preliminary results reported in this paper demonstrate the viability of the approach; yet training, feature selection and decoding methods need to be tailored better to the specific characteristics of the BCI signals obtained in these experiments.

\section{ACKNOWLEDGMENT}

We want to thank Claudia and Ilona Brandt for their support and commitment to this research.

\section{REFERENCES}

[1] A. B. Schwartz, X. T. Cui, D. J. Weber, and D. W. Moran, "Braincontrolled interfaces: movement restoration with neural prosthetics," Neuron, vol. 52, no. 1, pp. 205-220, 2006.

[2] J. P. Donoghue, "Bridging the brain to the world: a perspective on neural interface systems," Neuron, vol. 60, no. 3, pp. 511-521, 2008.

[3] M. A. Nicolelis and M. A. Lebedev, "Principles of neural ensemble physiology underlying the operation of brain-machine interfaces," Nature Reviews Neuroscience, vol. 10, no. 7, pp. 530-540, 2009.

[4] A. M. Green and J. F. Kalaska, "Learning to move machines with the mind," Trends in neurosciences, vol. 34, no. 2, pp. 61-75, 2011

[5] V. Gilja, C. Chestek, I. Diester, J. Henderson, K. Deisseroth, and K. Shenoy, "Challenges and opportunities for next-generation intracortically based neural prostheses," Biomedical Engineering, IEEE Transactions on, vol. 58, no. 7, pp. 1891-1899, 2011.
[6] L. Hochberg, D. Bacher, B. Jarosiewicz, N. Masse, J. Simeral, J. Vogel, S. Haddadin, J. Liu, S. Cash, P. van der Smagt et al., "Reach and grasp by people with tetraplegia using a neurally controlled robotic arm," Nature, vol. 485, no. 7398, pp. 372-375, 2012.

[7] J. L. Collinger, B. Wodlinger, J. E. Downey, W. Wang, E. C. TylerKabara, D. J. Weber, A. J. McMorland, M. Velliste, M. L. Boninger, and A. B. Schwartz, "High-performance neuroprosthetic control by an individual with tetraplegia," The Lancet, 2012.

[8] A. T. Duchowski, B. Pelfrey, D. H. House, and R. Wang, "Measuring gaze depth with an eye tracker during stereoscopic display," in Proceedings of the ACM SIGGRAPH Symposium on Applied Perception in Graphics and Visualization, ser. APGV '11. New York, NY, USA: ACM, 2011, pp. 15-22.

[9] J. Vogel, C. Castellini, and P. v. d. Smagt, "EMG-based teleoperation and manipulation with the DLR LWR-III," in IEEE/RSJ International Conference on Intelligent Robots and System, 2011, pp. 672-678.

[10] S. Ferguson and G. R. Dunlop, "Grasp recognition from myoelectric signals," in Proceedings of the Australasian Conference on Robotics and Automation, Auckland, New Zealand, 2002, pp. 83-87.

[11] A. Chan and K. Englehart, "Continuous myoelectric control for powered prostheses using hidden markov models," Biomedical Engineering, IEEE Transactions on, vol. 52, no. 1, pp. 121-124, 2005.

[12] P. A. Parker, K. Englehart, and B. Hudgins, "Myoelectric signal processing for control of powered limb prostheses," Journal of electromyography and kinesiology, vol. 16, no. 6, pp. 541-548, 2006.

[13] S. Bitzer and P. van der Smagt, "Learning EMG control of a robotic hand: towards active prostheses," in Proceedings IEEE International Conference on Robotics and Automation, 2006, pp. 2819-2823.

[14] C. Castellini and P. van der Smagt, "Surface EMG in advanced hand prosthetics," Biological Cybernetics, vol. 100, no. 1, pp. 35-47, 2008.

[15] P. K. Artemiadis and K. J. Kyriakopoulos, "EMG-based control of a robot arm using low-dimensional embeddings," IEEE Transactions on Robotics, vol. 26, no. 2, pp. 393-398, 2009.

[16] - "An EMG-based robot control scheme robust to time-varying EMG signal features," IEEE Transactions on Information Technology in Biomedicine, vol. 14, no. 3, pp. 582-588, 2010.

[17] — - "A switching regime model for the EMG-based control of a robot arm," IEEE Transactions on Systems, Man, and CyberneticsPart B: Cybernetics, vol. 41, no. 1, pp. 53-63, 2011.

[18] A. R. Marathe and D. M. Taylor, "Decoding position, velocity, or goal: does it matter for brain-machine interfaces?" Journal of neural engineering, vol. 8, no. 2, p. 025016, Apr. 2011.

[19] A. Savitzky and M. J. Golay, "Smoothing and differentiation of data by simplified least squares procedures." Analytical chemistry, vol. 36, no. 8, pp. 1627-1639, 1964.

[20] A. Albu-Schäffer, C. Ott, and G. Hirzinger, "A unified passivitybased control framework for position, torque and impedance control of flexible joint robots," The International Journal of Robotics Research, vol. 26, no. 1, pp. 23-39, 2007.

[21] A. Albu-Schäffer, S. Haddadin, C. Ott, A. Stemmer, T. Wimböck, and G. Hirzinger, "The DLR lightweight robot: design and control concepts for robots in human environments," Industrial Robot: An International Journal, vol. 34, no. 5, pp. 376-385, 2007.

[22] H. Liu, K. Wu, P. Meusel, N. Seitz, G. Hirzinger, M. Jin, Y. Liu, S. Fan, T. Lan, and Z. Chen, "Multisensory five-finger dexterous hand: The DLR/HIT hand II," in IEEE/RSJ International Conference on Intelligent Robots and System. IEEE, 2008, pp. 3692-3697.

[23] S. Haddadin, S. Parusel, R. Belder, J. Vogel, T. Rokahr, A. AlbuSchäffer, and G. Hirzinger, "Holistic design and analysis for the human-friendly robotic co-worker," in Intelligent Robots and Systems (IROS), 2010 IEEE/RSJ International Conference on. IEEE, 2010, pp. 4735-4742.

[24] S. Haddadin, M. Suppa, S. Fuchs, T. Bodenmüller, A. Albu-Schäffer, and G. Hirzinger, "Towards the robotic co-worker," Robotics Research, pp. 261-282, 2011.

[25] S. Haddadin, A. Albu-Schaffer, A. De Luca, and G. Hirzinger, "Collision detection and reaction: A contribution to safe physical humanrobot interaction," in Intelligent Robots and Systems, 2008. IROS 2008. IEEE/RSJ International Conference on. Ieee, 2008, pp. 3356-3363.

[26] D. W. Fellner, J. Behr, and U. Bockholt, "Instantreality-a framework for industrial augmented and virtual reality applications," in The 2nd Sino-German Workshop" Virtual Reality \& Augmented Reality in Industry": Invited Paper Proceedings. Shanghai Jiao Tong University, 2009, pp. $78-83$. 